https://helda.helsinki.fi

\title{
Intrauterine contraception after medical abortion : factors affecting success of early insertion
}

\section{Pohjoranta, Elina}

2017-03

Pohjoranta , E, Suhonen, S, Mentula , M \& Heikinheimo, O 2017 , ' Intrauterine contraception after medical abortion : factors affecting success of early insertion ' , Contraception , vol. 95 , no. 3 , pp. 257-262 . https://doi.org/10.1016/j.contraception.2016.10.012

http://hdl.handle.net/10138/297989

https://doi.org/10.1016/j.contraception.2016.10.012

publishedVersion

Downloaded from Helda, University of Helsinki institutional repository.

This is an electronic reprint of the original article.

This reprint may differ from the original in pagination and typographic detail.

Please cite the original version. 


\title{
Intrauterine contraception after medical abortion: factors affecting success of early insertion ${ }^{2}$, 论抎,
}

\author{
Elina Pohjoranta $^{\mathrm{a}}$, Satu Suhonen ${ }^{\mathrm{b}}$, Maarit Mentula ${ }^{\mathrm{a}}$, Oskari Heikinheimo ${ }^{\mathrm{a}, *}$ \\ ${ }^{a}$ Department of Obstetrics and Gynecology, University of Helsinki and Helsinki University Hospital, Helsinki, Finland \\ ${ }^{\mathrm{b}}$ Centralized Family Planning, Department of Social Services and Health Care, City of Helsinki, Helsinki, Finland
}

Received 8 July 2016; revised 28 October 2016; accepted 31 October 2016

\begin{abstract}
Objective: To assess the success and factors affecting early intrauterine device (IUD) provision after first trimester medical termination of pregnancy (MTOP).

Study design: Subgroup analysis of a randomized contraceptive trial assessing the long-term effects of early provision of intrauterine contraception following abortion. Altogether, 606 women undergoing MTOP were included and followed for 3 months. The intervention group $(n=307)$ was offered an IUD (either the levonorgestrel-releasing intrauterine system or copper-IUD) at a follow-up visit $1-4$ weeks after MTOP. The control group $(n=299)$ contacted primary health care for follow-up and contraceptive provision. Adverse events (infections, bleeding, residual tissue and incomplete abortion) were analyzed on intention-to-treat basis and IUD expulsions on per-protocol (PP) basis. Results: In the intervention group, 234 women (76.2\%) received the IUD as scheduled, 46 later (altogether 91.2\%). In the control group, the corresponding figures were $8(2.7 \%)$ and 64 [altogether $24.1 \%$, Odds ratio (OR) $(95 \%$ Confidence interval $(\mathrm{CI}))=32.7(20.3-52.6)$ ]. Eighty-five $(27.7 \%)$ women in the intervention group and $38(12.7 \%)$ in the control group received treatment (administration of antibiotics, misoprostol or surgical evacuation) because of presumed adverse event [2.63 (1.72-4.01)], mainly residual tissue. In the control group, 23 (60.5\%) of these occurred during the first 2 weeks. IUD expulsion occurred in 12 (5.4\%) of the 222 women in the intervention group (PP basis).

Conclusions: When provided as part of abortion service, most early insertions following MTOP were performed as planned. The main reason for postponement was overdiagnosis of adverse events suspected at follow-up. The rate of IUD expulsion was similar to that reported previously.

Implications: Early insertion following MTOP is safe, and the rate of IUD expulsion is low. Most adverse events possibly delaying IUD insertion occur early. Based on timing of adverse events in the control group, IUD insertion at approximately 2 weeks after completed MTOP seems optimal. (C) 2017 Elsevier Inc. All rights reserved.
\end{abstract}

Keywords: Medical abortion; Complication; Adverse event; Intrauterine contraception; IUD expulsion

\footnotetext{
Funding: This study was supported by Helsinki University Central Hospital Research funds and research grants provided by the Antti and Jenny Wihuri Foundation and the Yrjö Jahnsson Foundation. The sponsors had no role in the planning or carrying out of the study or in analyzing the study results. 放败 Declaration of interests: $\mathrm{OH}$ has served on advisory boards for Bayer Healthcare, Gedeon Richter and MSD Finland (part of Merck \& Co. Inc.) and designed and lectured at educational events of these companies. SS has served as an advisor for Exeltis and Gedeon Richter and lectured at educational events of Bayer and MSD Finland (part of Merck \& Co. Inc.). The other authors have no conflicts of interests to declare.

$\star$ Clinical trial registration: Registered at www.clinicaltrials.gov (NCT01223521).

* Corresponding author.

E-mail address: oskari.heikinheimo@helsinki.fi (O. Heikinheimo).
}

\section{Introduction}

Several cohort studies have shown that postabortion use of long-acting reversible methods of contraception, and especially that of intrauterine contraceptive devices (IUDs), is associated with reduced incidence of recurrent unintended pregnancy [1-4].

According to international guidelines, an IUD can be safely inserted at the time of surgical termination of pregnancy (TOP) or after medical termination of pregnancy (MTOP) when, with reasonable certainty, the woman is no longer pregnant [5-7]. Immediate insertion of an IUD at the time of surgical TOP leads to a higher overall rate of use compared with delayed insertion $[8,9]$. This is explained in part by the high percentage of women not attending the scheduled follow-up visit $[4,10]$. 
During the last few decades, MTOP has become the dominant method of early abortion in several countries. In 2014, 95\% of all induced abortions were medical in Finland, and the figure was 51\% in England and Wales [11,12]. In 2015, the figures were $91 \%$ in Sweden, $88 \%$ in Norway and $81 \%$ in Scotland [13-15]. In the US, the corresponding figure in 2011 was $23 \%$ [16]. MTOP is associated with a rate of up to $20 \%$ of early adverse events, some of which may interfere with the initiation of intrauterine contraception [17], although in several studies, early IUD insertion between 1 to 4 weeks following MTOP has proven to be safe and effective $[1,18-20]$. However, the overall success of IUD provision following MTOP and the factors affecting it have not been assessed in large-scale trials.

We have undertaken a randomized controlled clinical trial (RCT) evaluating the long-term effects of routine provision of IUD [either the levonorgestrel-releasing intrauterine system (LNG-IUS) or a copper IUD (Cu-IUD)] in association with first-trimester TOP versus the practice of initiating oral contraceptives at the time of TOP with the plan of later provision of IUD [21]. We found that the incidence of recurrent unintended pregnancy was significantly reduced among women randomized to early IUD provision (intervention group) already during the first year after the index abortion [21].

The present work is a subgroup analysis of the above-mentioned RCT. We assessed the success of early (i.e., between 1 and 4 weeks) initiation of intrauterine contraception following first-trimester MTOP and the factors that may interfere with it. Second, we analyzed all adverse events and complications - those related to MTOP itself (infection, heavy bleeding and incomplete abortion) and their timing and those related to IUD insertion (infection, expulsion, unscheduled visits) within 3 months after MTOP.

\section{Materials and methods}

\subsection{Study population}

This study was performed in collaboration with Helsinki University Hospital and the City of Helsinki. The inclusion criteria were residence in Helsinki, age $\geq 18$ years, first-trimester pregnancy with desire to terminate it, acceptance of intrauterine contraception and willingness to participate and to sign the informed consent form. The exclusion criteria were contraindications to intrauterine contraception, such as uterine anomaly, acute genital infection or cytological findings in Pap tests requiring further investigation.

The study protocol has been described in detail previously [21]. In brief, in the primary study, 751 women were randomized into two groups to assess the effects of intrauterine contraception initiated in association with TOP [either medical $(n=606)$ or surgical $(n=142)$ ] on the need of subsequent TOP. Three women decided to continue with the pregnancy after randomization.

Following MTOP, the intervention group received an IUD at a follow-up visit including clinical and ultrasonographic examination at $1-4$ weeks after MTOP. A follow-up visit was scheduled at 3 months after TOP to ensure acceptance of the method and correct location of the IUD by visualizing the strings. If the strings were not detected or appeared to be too long, vaginal ultrasonography was arranged.

Women in the control group received oral contraceptives for immediate postabortion contraception. They were advised to schedule a follow-up visit at 2-4 weeks to verify the outcome of TOP (serum hCG measurement) and for further contraceptive provision (including IUD insertion) at the primary healthcare services of the City of Helsinki. The IUD insertions occurring within 3 months after the abortion were verified using the electronic patient files of the City of Helsinki.

All procedures, including follow-up of the control group, were performed according to Finnish guidelines on induced abortion [22]. All women were screened for Chlamydia trachomatis infection prior to TOP.

All study participants were advised to contact the study hospital in cases of diagnosed or suspected TOP-related complications. The follow-up visits including the planned IUD insertions were performed by the study physicians. Women with unscheduled hospital visits were mainly examined by physicians on duty. The study hospital is in charge of the abortion care and emergency gynecological services in the Helsinki metropolitan area. In addition, the electronic database of the hospital is comprehensive and covers all medical specialties.

All additional visits to the hospital within 3 months after TOP were evaluated and analyzed. Unsuccessful MTOP was defined as continuing pregnancy with a living fetus. Cases of missed abortion or suspected residual tissue were analyzed according to the type of treatment provided (surgical evacuation, administration of misoprostol or expectant management). Clinically diagnosed genital infections were defined by purulent discharge and/or uterine tenderness with or without fever and classified into those treated with oral or intravenous antibiotics.

Complete IUD expulsion was defined as a totally expelled device and partial expulsion as IUD displacement of more than $15 \mathrm{~mm}$ from the uterine fundus in vaginal ultrasonography. A new device was offered in all cases of IUD expulsion.

\subsection{Ethics approval}

The study was approved by the Ethics Committee of the Hospital District of Helsinki and Uusimaa (HUS 260/13/03/ 03/2009), as well as the City of Helsinki (10-1138/054), and it was registered at www.clinicaltrials.gov (NCT01223521).

\subsection{Statistical analysis}

Adverse events and complications after MTOP and their timing were analyzed on an intention-to-treat (ITT) basis. Assessment of complications related to early provision of an IUD (up to 4 weeks) was performed on a per-protocol (PP) basis. Statistical analyses were performed using IBM SPSS statistics software Version 23.0 (IBM Corp., Armonk, NY, USA). The chi-square test was used to evaluate possible differences in the incidence of various complications 
between the two groups. Logistic regression was used to calculate odds ratios (ORs) with a 95\% confidence interval (95\% CI). To analyze the cumulative survival ratios regarding timing of complications or adverse events, the Kaplan-Meier model was used.

\section{Results}

Altogether 606 women undergoing MTOP (Fig. 1) were included in the present analysis. In 378 cases (62.4\%) MTOP was performed using self-administration of misoprostol at home. The characteristics of the participants are presented in detail in Table 1.

In the intervention group, a total of $280(91.2 \%)$ women received an IUD, whereas the corresponding number in the control group was $72[24.1 \%$, OR $(95 \%$ CI $)=32.7$ (20.3-52.6)]. The majority of the intervention group $[n=234$ (76.2\%)] had an IUD insertion within 4 weeks after MTOP, as planned initially. Later insertions $[n=46(15.0 \%)]$ were due to suspected residual tissue and/or infection $(n=43)$ or nonattendance at the initially scheduled visit $(n=3)$. In the control group altogether, $8(2.7 \%)$ women received the IUD within 4 weeks and $64(21.4 \%)$ between 1 and 3 months. The differences in comparison to the intervention group were significant [OR $(95 \% \mathrm{CI})=116.6(55.1-246.8)$ and $0.647(0.426-0.982)$, respectively].

Twelve women in the intervention group received an IUD in association with surgical evacuation because of residual tissue and were therefore excluded from the PP analysis. In the intervention group, 222 women and, in the control group, 297 women were included in the PP analysis. Of the 222 women in the intervention group, $204(91.9 \%)$ chose the LNG-IUS and, 18 (8.1\%), the Cu-IUD.
Table 1

Demographics of the study groups (ITT basis). The data are reported as $n(\%)$ unless stated other vice.

\begin{tabular}{|c|c|c|c|}
\hline Variable & $\begin{array}{l}\text { Intervention } \\
\text { group }(n=307)\end{array}$ & $\begin{array}{l}\text { Control } \\
\text { group }(n=299)\end{array}$ & p-value \\
\hline Age [years (mean, SD)] & $27.7(6.40)$ & $28.1(6.51)$ & 0.40 \\
\hline \multicolumn{4}{|l|}{ Marital status } \\
\hline Married & 58 (18.9) & $42(14.0)$ & 0.21 \\
\hline Cohabitating & $83(27.0)$ & $78(26.1)$ & \\
\hline Single & $166(54.1)$ & $179(59.9)$ & \\
\hline Regular/Daily smoking & $153(49.8)$ & $152(51.0)$ & 0.77 \\
\hline $\begin{array}{l}\text { Chlamydia trachomatis at the time } \\
\text { of abortion }\end{array}$ & $8(2.6)$ & $6(2.0)$ & 0.62 \\
\hline $\begin{array}{l}\text { Duration of pregnancy at the time } \\
\text { of abortion [days (mean, SD)] }\end{array}$ & $57.5(11.1)$ & $56.0(11.3)$ & 0.09 \\
\hline$\leq 42$ days & $14(4.6)$ & $17(5.7)$ & 0.73 \\
\hline 43-63 days & $215(70.0)$ & $212(70.9)$ & \\
\hline $64-84$ days & $78(25.4)$ & $70(23.4)$ & \\
\hline \multicolumn{4}{|l|}{ Previous pregnancies } \\
\hline Primigravid & 98 (31.9) & $100(33.4)$ & 0.69 \\
\hline History of induced abortion(s) & $139(45.3)$ & $120(40.3)$ & 0.21 \\
\hline Parous & $148(48.2)$ & $133(44.6)$ & 0.38 \\
\hline
\end{tabular}

\subsection{Postabortion adverse events and complications and} their timing

During the 3-month follow-up period, 43 women had an unscheduled visit (Table 2) because of bleeding [20 (6.5\%) in the intervention group and $23(7.7 \%)$ in the control group, $\mathrm{p}=.572]$. Heavy bleeding was treated by means of surgical evacuation in $9(2.9 \%)$ versus $11(3.7 \%)$ women, respectively $(\mathrm{p}=.607)$. However, only $5(0.8 \%)$ women received blood transfusion; $[2(0.7 \%)$ in the intervention group and $3(1.0 \%)$ in the control group, $\mathrm{p}=.632]$. Nineteen women $(45.2 \%)$ contacted the hospital because of bleeding within 14 days after MTOP (6 in the intervention group vs. 13 in the control group).

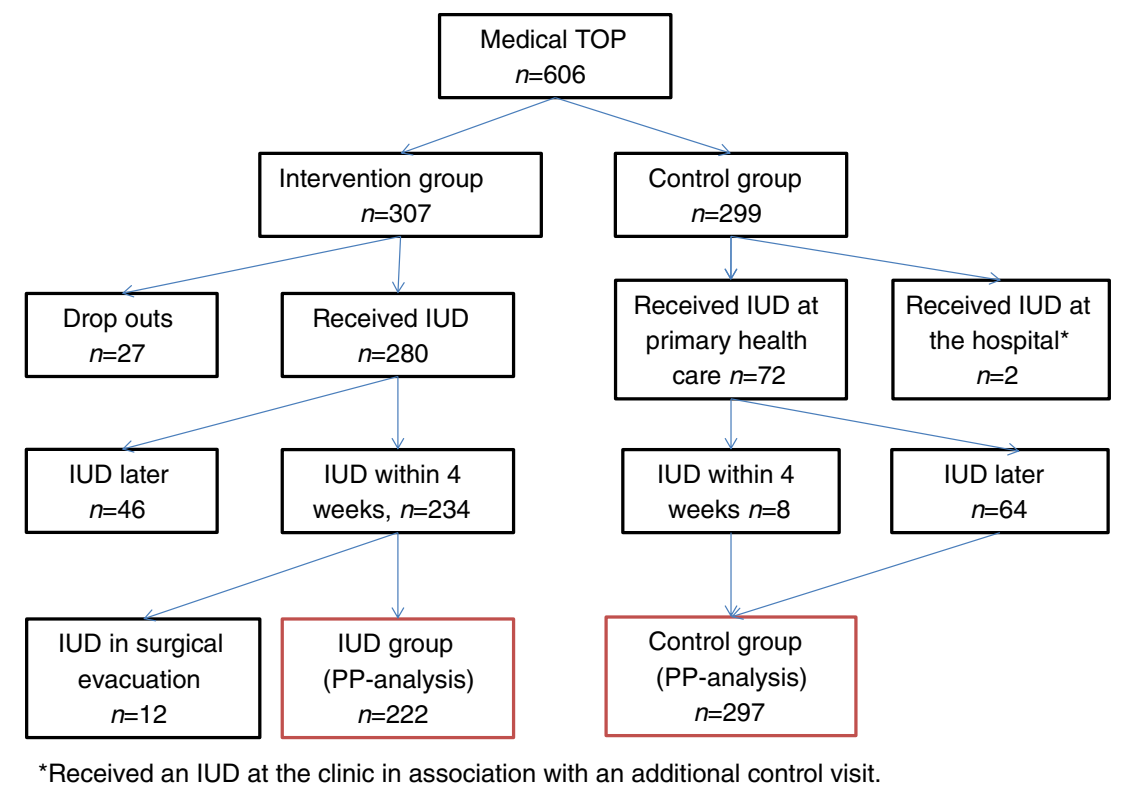

Fig. 1. Flow chart of the study subjects. 
Table 2

Adverse events/complications within 3 months after MTOP in the study groups (ITT basis). The data are reported as $n$ (\%) unless stated other vice.

\begin{tabular}{|c|c|c|c|}
\hline Variable & Intervention group $(n=307)$ & Control group $(n=299)$ & OR $(95 \% \mathrm{CI})$ \\
\hline Adverse event/complication judged to require intervention & $85(27.7)$ & $38(12.7)$ & $2.63(1.72-4.01)$ \\
\hline Intervention within 14 days after abortion & $20(6.5)$ & $23(7.7)$ & $0.84(0.45-1.56)$ \\
\hline Intervention between 2 and 4 weeks (15-28 days) & $59(19.2)$ & $7(2.3)$ & $9.92(4.45-22.12)$ \\
\hline Intervention between 4 and 6 weeks (29-42 days) & $5(1.6)$ & $6(2.0)$ & $0.81(0.24-2.68)$ \\
\hline Intervention between 6 weeks and 3 months ( $43-90$ days) & $1(0.3)$ & $2(0.7)$ & $0.49(0.04-5.38)$ \\
\hline Extra visit due to bleeding & $20(6.5)$ & $23(7.7)$ & $0.83(0.45-1.56)$ \\
\hline Heavy bleeding & $11(3.6)$ & $15(5.0)$ & $0.70(0.32-1.56)$ \\
\hline - treated by blood transfusion & $2(0.7)$ & $3(1.0)$ & $0.65(0.11-3.90)$ \\
\hline - treated by surgical evacuation & $9(2.9)$ & $11(3.7)$ & $0.79(0.32-1.94)$ \\
\hline Time between the abortion and visit due to/dg of bleeding [days, (mean $\pm \mathrm{SD})$ ] & $16.2(12.7)$ & $19.2(19.3)$ & \\
\hline Infection & $29(9.4)$ & $17(5.7)$ & $1.73(0.93-3.22)$ \\
\hline - treated by intravenous antibiotics & $10(3.3)$ & $9(3.0)$ & $1.08(0.43-2.71)$ \\
\hline - treated by per oral antibiotics & $19(6.2)$ & $8(2.7)$ & $2.40(1.03-5.57)$ \\
\hline Time between the abortion and diagnosis of/visit due to infection [days, (mean $\pm \mathrm{SD}$ )] & $16.8(9.6)$ & $15.8(15.3)$ & \\
\hline Residual tissue & $72(23.5)$ & $30(10.0)$ & $2.75(1.73-4.35)$ \\
\hline - treated by means of surgical evacuation & $40(13.0)$ & $24(8.0)$ & $1.72(1.01-2.93)$ \\
\hline - treated by means of additional misoprostol & $52(16.9)$ & $9(3.0)$ & $6.57(3.18-13.60)$ \\
\hline - waiting & $4(1.3)$ & $4(1.3)$ & $0.97(0.24-3.93)$ \\
\hline Treatment within 14 days & $14(19.4)$ & $18(60.0)$ & $0.75(0.36-1.53)$ \\
\hline $2-4$ weeks & $55(76.4)$ & $7(23.3)$ & $9.10(4.07-20.35)$ \\
\hline 4-6 weeks & $3(4.2)$ & $4(13.3)$ & $0.73(0.16-3.28)$ \\
\hline 6 weeks -3 months & 0 & $1(3.3)$ & - \\
\hline Time between abortion and visit due to/diagnosis of residual tissue [days, (mean $\pm \mathrm{SD}$ )] & $17.1(6.7)$ & $14.9(12.9)$ & \\
\hline Missed abortion & $2(0.7)$ & $4(1.3)$ & $0.48(0.09-2.66)$ \\
\hline Ongoing pregnancy & $1(0.3)$ & 0 & - \\
\hline
\end{tabular}

During the 3-month follow-up period, 29 (9.4\%) women in the intervention group and $17(5.7 \%)$ in the control group received antibiotics because of clinically diagnosed infection ( $\mathrm{p}=.081)$ (Fig. 2A). Infection treated with intravenous antibiotics occurred in $10(3.3 \%)$ and $9(3.0 \%)$ cases in the intervention and control groups, respectively $(\mathrm{p}=.861)$. There were $19(6.2 \%)$ and $8(2.7 \%)$ cases of infection treated with oral antibiotics in the intervention and control groups ( $\mathrm{p}=.036)$, respectively.

Residual tissue was suspected in $72(23.5 \%)$ and $30(10.0 \%)$ cases in the intervention and control groups, respectively $(\mathrm{p}<.001)$ (Fig. 2B). Treatment with additional misoprostol was performed in $52(16.9 \%)$ versus $9(3.0 \%)$ women $(\mathrm{p}<.001)$. Of the cases of suspected residual tissue, $14(19.4 \%)$ in the intervention group and $18(60.0 \%)$ in the control group were detected within 14 days after TOP. Surgical evacuation of residual tissue was performed in $40(13.0 \%)$ versus $24(8.0 \%)$ cases, respectively $(\mathrm{p}=.045)$. The cumulative proportions of women who did not undergo surgical evacuation were $79.7 \%$ versus $84.2 \%, \mathrm{p}=.032$.

\subsection{Complications associated with IUD insertion}

There were no cases of uterine perforation or bleeding requiring blood transfusion related to IUD insertion in the intervention group. Five women received antibiotics after IUD insertion, one of whom received the IUD 2 weeks after MTOP during surgical evacuation of residual tissue. She was later diagnosed with persistent residual tissue and was treated by surgical reevacuation and intravenous antibiotics 1 week after IUD insertion.

During the 3-month follow-up period, there were 12 IUD expulsions in all $(12 / 222$; i.e., $5.4 \%)$ among women who received the IUD according to the study protocol in the intervention group (PP analysis). Of these, 7 were partial and 5 total. We have no data concerning the possible IUD expulsions in the control group.

Three women belonging to the control group that had been fitted with an IUD returned to the hospital for consultation during the 3-month follow-up, two because of bleeding and one because of pain.

Analysis of the potential risk factors of IUD expulsion in the intervention group revealed that uterine sound measure, previous pregnancy or parity and body mass index did not correlate with the incidence of expulsion. Longer duration of gestation was associated with an elevated risk of expulsion. Surprisingly, thickness of intrauterine content prior to IUD insertion seemed to inversely correlate with the rate of expulsion (Supplementary Table 3).

\section{Discussion}

We found that when provided as part of the abortion service, more than $75 \%$ of the planned early IUD insertions were carried out as scheduled following first trimester MTOP. Including the cases with a minor delay, the figure was more than $90 \%$. Approximately $15 \%$ of the planned insertions were rescheduled, 


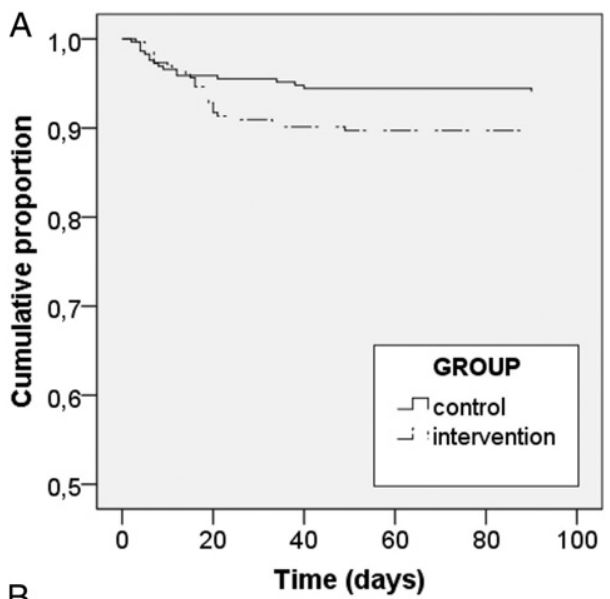

B

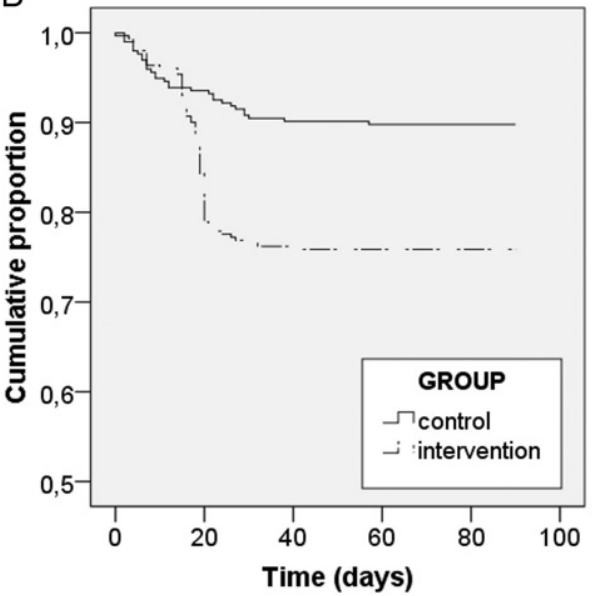

Fig. 2. (A) Cumulative proportion of subjects in both study groups without infection. (B) Cumulative proportion of subjects in both study groups without suspected residual tissue.

most commonly because of suspected residual tissue observed in ultrasonography. The rates of expulsions $(5 \%)$ or complications following early IUD insertion were low and similar to those reported following insertion at the time of surgical TOP [23]. However, when the IUD was provided at the primary health care, insertion within 3 months took place in only $24 \%$ of the cases. The reasons for the difference are likely to be associated with both patient and healthcare system-related factors.

The goal of the primary RCT is to compare the long-term (5-year follow-up) effects of routine provision of an IUD at the time of TOP to the current practice of postabortion contraception. Therefore, also the early follow-up protocol was different. Nevertheless, the randomized design of the study with different follow-up protocols also proved to be a strength regarding diagnosis and treatment of adverse events addressed in the present subanalysis. In addition, we were reliably able to assess all complications requiring hospital-based medical attention.

The shortcomings of the study include the fact that the diagnostic criteria for the various adverse events were variable. In addition, other than IUD insertions, we had limited information concerning the care at the primary health care. Moreover, the study was designed as a contraceptive trial and powered according to the expected rate of subsequent TOP during the follow-up period. Therefore, rare outcomes such as uterine perforation cannot be reliably assessed.

As observed previously [17], a high number of minor adverse events following first-trimester MTOP was seen in the present study, too. In addition, the rate of complications treated by surgical evacuation, intravenous antibiotics or blood transfusion was relatively high $(19.9 \%$ in the intervention group and $15.7 \%$ in the control group; $\mathrm{p}=.182$ ). Corresponding figures derived from Finnish national health registries revealed considerably lower rate of postabortion surgical evacuation and use of antibiotics [17]. The high rate of postabortion interventions seen in the present study is likely to be associated with the investigational nature of the study, with scheduled additional study visits in the intervention group and overall easier access to additional consultation.

Follow-up practices vary after early MTOP. Based on the results of a randomized study by Fiala et al., Finnish guidelines on induced abortion recommend verification of abortion outcome by means of serum hCG measurement $2-3$ weeks after TOP $[22,24]$. In the present study, women randomized to the control group underwent the recommended follow-up in primary healthcare, whereas the intervention group attended the IUD insertion visit at 1-4 weeks as well as the 3-month check-up visit at the study hospital.

As reported previously, the use of ultrasonography at the follow-up visit significantly increased diagnosis of suspected (and most likely clinically meaningless) residual tissue [25]. This was seen despite the fact that all examinations were carried out by investigators with significant experience in MTOP. The use of ultrasonography resulted in an increased rate of intervention, especially administration of misoprostol, in the intervention group. It is likely that many of these treatments were unnecessary, potentially causing harm for these women. Thus, even if ultrasonography is useful when missed abortion or on-going pregnancy is suspected, routinely performing ultrasound shortly after MTOP can lead to inaccurate diagnostic impressions [25,26].

In addition to the above, the diagnosis of genital infections and subsequent use of orally administered antibiotics was higher in the intervention group. In this group, one third of the infections were diagnosed before and half at the planned IUD insertion visit. However, the number of women receiving intravenous antibiotics was similar in the two groups.

The rate of IUD expulsion (most cases of which were partial) during the 3-month follow-up period was $5.4 \%$. Somewhat higher figures (7-11\%) during 6 months of follow-up have been reported following early (1-4 weeks) insertion after early medical abortion $[18,27]$. The corresponding figure following IUD insertion at the time of surgical evacuation has been $5 \%$ [23]. Thus, early IUD insertion after MTOP is associated with a rate of expulsion similar to that after insertion at the time of surgical TOP.

With the small number of expulsions in our study, we have limited power to make conclusions concerning the risk factors of expulsion. However, our findings are in agreement 
with those of Sääv et al. concluding that endometrial ultrasonographic findings have a poor predictive value in estimating the risk of IUD expulsion following MTOP [18].

Our findings, as well as those reported previously [18,20], support a pragmatic approach to early IUD provision after MTOP. Following verification of complete abortion (preferably by hCG measurement) in the absence of clinically diagnosed complications, an IUD can be safely inserted at $1-4$ weeks after MTOP. Provision of IUD as an integral part of abortion care and by the same organization providing the abortion seems beneficial. Based on the timing of complications observed, especially in the control group, IUD insertion within 2 weeks after TOP may be optimal. On the other hand, a delay in initiation of contraception is associated with a higher rate of nonattendance at follow-up visits as well as risk of future unintended pregnancy $[20,21]$. Thus, early time-points may also be important in order to optimize compliance as regards contraceptive initiation and continuation. Overall, the data encourage the development of well-coordinated fast-tract services aimed at early provision of intrauterine contraception following MTOP.

Supplementary data to this article can be found online at http://dx.doi.org/10.1016/j.contraception.2016.10.012.

\section{Acknowledgements}

This study was supported by Helsinki University Central Hospital Research funds and research grants provided by the Antti and Jenny Wihuri Foundation and the Yrjö Jahnsson Foundation. The sponsors had no role in the planning or carrying out of the study or in analyzing the study results. Study nurse Pirjo Ikonen is gratefully thanked for her irreplaceable contribution to this study.

\section{References}

[1] Heikinheimo O, Gissler M, Suhonen S. Age, parity, history of abortion and contraceptive choices affect the risk of repeat abortion. Contraception 2008;78:149-54.

[2] Peipert JF, Madden T, Allsworth JE, Secura GM. Preventing unintended pregnancies by providing no-cost contraception. Obstet Gynecol 2012;120:1291-7.

[3] Rose SB, Lawton BA. Impact of long-acting reversible contraception on return for repeat abortion. Obstet Gynecol 2012;206:37.e1-6.

[4] Cameron ST, Berugoda N, Johnstone A, Glasier A. Assessment of a 'fasttrack' referral service for intrauterine contraception following early medical abortion. J Fam Plann Reprod Health Care 2012;38:175-8.

[5] Grimes DA, Lopez LM, Schulz KF, Stanwood NL. Immediate postabortal insertion of intrauterine devices. Cochrane Database Syst Rev 2010:CD001777.

[6] Okusanya BO, Oduwole O, Effa EE. Immediate postabortal insertion of intrauterine devices. Cochrane Database Syst Rev 2014;7:CD001777.
[7] Safe abortion: technical and policy guidance for health systems. 2nd ed. WHO; 2012 [Geneva], http://www.who.int/reproductivehealth/ publications/unsafe_abortion/9789241548434/en/.

[8] Goodman S, Hendlish SK, Reeves MF, Foster-Rosales A. Impact of immediate postabortal insertion of intrauterine contraception on repeat abortion. Contraception 2008;78:143-8.

[9] Fox MC, Oat-Judge J, Severson K, Jamshidi RM, Singh RH, McDonald-Mosley $\mathrm{R}$, et al. Immediate placement of intrauterine devices after first and second trimester pregnancy termination. Contraception 2011;83:34-40.

[10] Pohjoranta E, Suhonen S, Heikinheimo O. Attendance at post-abortal follow-up visits is low - can the risks of non-attendance be identified? Acta Obstet Gynecol Scand 2011;90:543-6.

[11] Abortion statistics 2014. National Institute for Health and Welfare; 2015 [http://www.julkari.fi/handle/10024/127103, Last accessed: 8.6.2016].

[12] Abortion statistics, England and Wales 2014. Department of Health; 2015 [https://www.gov.uk/government/statistics/report-on-abortionstatistics-in-england-and-wales-for-2014, Last accessed: 8.6.2016].

[13] Abort - fakta med statistikk. Folkehelseinstituttet Norway; 2016 [http:// www.fhi.no/tema/abort/fakta-om-abort, Last accessed: 8.6.2016].

[14] Termination of pregnancy statistics. National Health Service Scotland, Information Services Division; 2016 [http://www.isdscotland.org/HealthTopics/Sexual-Health/Publications/index.asp\#1655, Last accessed: 8.6.2016].

[15] Statistik om aborter 2015. Socialstyrelsen; 2016. [http://www. socialstyrelsen.se/publikationer2016/2016-5-20, Last accessed: 8.6.2016]

[16] Induced abortion in the United States. Guttmacher Institute; 2016 [https://www.guttmacher.org/fact-sheet/induced-abortion-unitedstates, Last accessed: 8.6.2016].

[17] Niinimäki M, Pouta A, Bloigu A, Gissler M, Hemminki E, Suhonen S, et al. Immediate complications after medical compared with surgical termination of pregnancy. Obstet Gynecol 2009;114:795-804.

[18] Saav I, Stephansson O, Gemzell-Danielsson K. Early versus delayed insertion of intrauterine contraception after medical abortion - a randomized controlled trial. PLoS One 2012;7:e48948.

[19] Cameron S. Postabortal and postpartum contraception. Best Pract Res Clin Obstet Gynaecol 2014;28:871-80.

[20] Shimoni N, Davis A, Ramos ME, Rosario L, Westhoff C. Timing of copper intrauterine device insertion after medical abortion: a randomized controlled trial. Obstet Gynecol 2011;118:623-8.

[21] Pohjoranta E, Mentula M, Gissler M, Suhonen S, Heikinheimo O. Provision of intrauterine contraception in association with first trimester induced abortion reduces the need of repeat abortion: firstyear results of a randomized controlled trial. Hum Reprod 2015;30:2539-46.

[22] Current Care Guidelines: Abortion. The Finnish medical society Duodecim. http:/www.kaypahoito.fi/web/kh/suositukset/suositus?id=hoi27050 2013 [Last accessed: 29.6.2016].

[23] Bednarek PH, Creinin MD, Reeves MF, Cwiak C, Espey E, Jensen JT, et al. Immediate versus delayed IUD insertion after uterine aspiration. Med 2011;364:2208-17.

[24] Fiala C, Safar P, Bygdeman M, Gemzell-Danielsson K. Verifying the effectiveness of medical abortion; ultrasound versus hCG testing. Obstet Gynecol Reprod Biol 2003;109:190-5.

[25] Suhonen S, Heikinheimo O, Tikka M, Haukkamaa M. The learning curve is rapid in medical termination of pregnancy-first-year results from the Helsinki area. Contraception 2003;67:223-7.

[26] Reeves MF, Fox MC, Lohr PA, Creinin MD. Endometrial thickness following medical abortion is not predictive of subsequent surgical intervention. Ultrasound Obstet Gynecol 2009;34:104-9.

[27] Shimoni N, Davis A, Westhoff C. Can ultrasound predict IUD expulsion after medical abortion? Contraception 2014;89:434-9. 